\title{
The NANOGrav 11 yr Data Set: Limits on Gravitational Waves from Individual Supermassive Black Hole Binaries
}

K. Aggarwal ${ }^{1,2}$, Z. Arzoumanian ${ }^{3}$, P. T. Baker ${ }^{1,2}$ (D) A. Brazier ${ }^{4,5}$, M. R. Brinson ${ }^{6}$, P. R. Brook ${ }^{1,2}$, S. Burke-Spolaor ${ }^{1,2}$ (D),

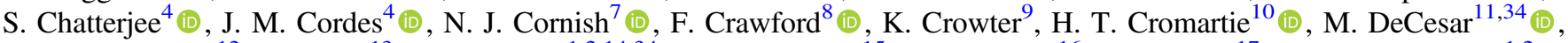
P. B. Demorest ${ }^{12}$, T. Dolch $^{13}$ (1) J. A. Ellis ${ }^{1,2,14,34}$, R. D. Ferdman ${ }^{15}$ (1), E. Ferrara ${ }^{16}$, E. Fonseca ${ }^{17}$, N. Garver-Daniels ${ }^{1,2}$, P. Gentile ${ }^{1,2}$ (1) , J. S. Hazboun ${ }^{18,34}$ (1) , A. M. Holgado ${ }^{19}$ (i), E. A. Huerta ${ }^{19}$, K. Islo $^{6}$, R. Jennings ${ }^{4}$, G. Jones ${ }^{20}$, M. L. Jones ${ }^{1,2}$ (1),

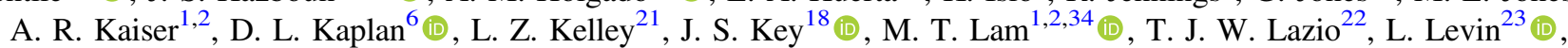

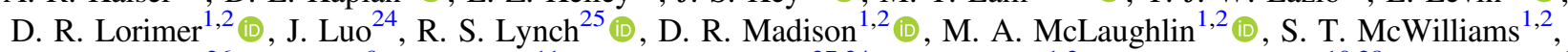

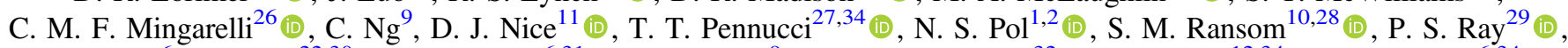

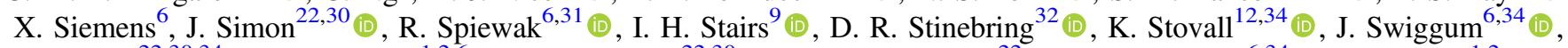

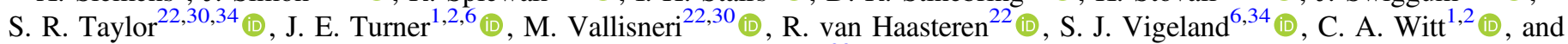
W. W. $\mathrm{Zhu}^{33}$ (1)

(The NANOGrav Collaboration) ${ }^{35}$

${ }^{1}$ Department of Physics and Astronomy, West Virginia University, P.O. Box 6315, Morgantown, WV 26506, USA

${ }^{2}$ Center for Gravitational Waves and Cosmology, West Virginia University, Chestnut Ridge Research Building, Morgantown, WV 26505, USA

${ }^{3}$ X-Ray Astrophysics Laboratory, NASA Goddard Space Flight Center, Code 662, Greenbelt, MD 20771, USA

${ }^{4}$ Department of Astronomy, Cornell University, Ithaca, NY 14853, USA ${ }^{5}$ Cornell Center for Advanced Computing, Ithaca, NY 14853, USA

${ }^{6}$ Center for Gravitation, Cosmology and Astrophysics, Department of Physics, University of Wisconsin-Milwaukee, P.O. Box 413, Milwaukee, WI 53201, USA vigeland@uwm.edu

${ }^{7}$ Department of Physics, Montana State University, Bozeman, MT 59717, USA

${ }^{8}$ Department of Physics and Astronomy, Franklin \& Marshall College, P.O. Box 3003, Lancaster, PA 17604, USA

${ }^{9}$ Department of Physics and Astronomy, University of British Columbia, 6224 Agricultural Road, Vancouver, BC V6T 1Z1, Canada

${ }^{10}$ University of Virginia, Department of Astronomy, P.O. Box 400325, Charlottesville, VA 22904, USA

${ }_{11}$ Department of Physics, Lafayette College, Easton, PA 18042, USA

${ }^{12}$ National Radio Astronomy Observatory, 1003 Lopezville Road, Socorro, NM 87801, USA

${ }^{13}$ Department of Physics, Hillsdale College, 33 E. College Street, Hillsdale, Michigan 49242, USA

${ }^{14}$ Infinia ML, 202 Rigsbee Avenue, Durham, NC 27701, USA

${ }^{15}$ Department of Physics, University of East Anglia, Norwich, UK

${ }^{16}$ NASA Goddard Space Flight Center, Greenbelt, MD 20771, USA

${ }^{17}$ Department of Physics, McGill University, 3600 University Street, Montreal, QC H3A 2T8, Canada

${ }^{18}$ University of Washington Bothell, 18115 Campus Way NE, Bothell, WA 98011, USA

${ }^{19}$ NCSA and Department of Astronomy, University of Illinois at Urbana-Champaign, Urbana, Illinois 61801, USA

${ }^{20}$ Department of Physics, Columbia University, New York, NY 10027, USA

${ }^{21}$ Center for Interdisciplinary Exploration and Research in Astrophysics (CIERA), Northwestern University, Evanston IL 60208, USA

22 Jet Propulsion Laboratory, California Institute of Technology, 4800 Oak Grove Drive, Pasadena, CA 91109, USA

${ }^{23}$ Jodrell Bank Centre for Astrophysics, University of Manchester, Manchester, M13 9PL, UK

${ }^{24}$ Center for Advanced Radio Astronomy, University of Texas Rio Grande Valley, Brownsville, TX 78520, USA

${ }^{25}$ Green Bank Observatory, P.O. Box 2, Green Bank, WV 24944, USA

${ }^{26}$ Center for Computational Astrophysics, Flatiron Institute, 162 Fifth Avenue, New York, NY 10010, USA

${ }^{27}$ Hungarian Academy of Sciences MTA-ELTE Extragalactic Astrophysics Research Group, Institute of Physics, Eötvös Loránd University, Pázmány P. s. 1/A, 1117 Budapest, Hungary

${ }^{28}$ National Radio Astronomy Observatory, 520 Edgemont Road, Charlottesville, VA 22903, USA

${ }^{29}$ Naval Research Laboratory, Washington DC 20375, USA

${ }^{30}$ Theoretical AstroPhysics Including Relativity (TAPIR), MC 350-17, California Institute of Technology, Pasadena, California 91125, USA

${ }^{31}$ Centre for Astrophysics and Supercomputing, Swinburne University of Technology, PO Box 218, Hawthorn, VIC 3122, Australia

${ }^{32}$ Department of Physics and Astronomy, Oberlin College, Oberlin, OH 44074, USA

${ }^{33}$ CAS Key Laboratory of FAST, Chinese Academy of Science, Beijing 100101, People's Republic of China Received 2018 December 28; revised 2019 April 17; accepted 2019 May 15; published 2019 July 31

\begin{abstract}
Observations indicate that nearly all galaxies contain supermassive black holes at their centers. When galaxies merge, their component black holes form SMBH binaries (SMBHBs), which emit low-frequency gravitational waves (GWs) that can be detected by pulsar timing arrays. We have searched the North American Nanohertz Observatory for Gravitational Waves 11 yr data set for GWs from individual SMBHBs in circular orbits. As we did not find strong evidence for GWs in our data, we placed 95\% upper limits on the strength of GWs from such sources. At $f_{\mathrm{gw}}=8 \mathrm{nHz}$, we placed a sky-averaged upper limit of $h_{0}<7.3(3) \times 10^{-15}$. We also developed a technique to determine the significance of a particular signal in each pulsar using "dropout" parameters as a way of identifying spurious signals. From these upper limits, we ruled out SMBHBs emitting GWs with $f_{\mathrm{gw}}=8 \mathrm{nHz}$ within $120 \mathrm{Mpc}$ for $\mathcal{M}=10^{9} M_{\odot}$, and within $5.5 \mathrm{Gpc}$ for $\mathcal{M}=10^{10} M_{\odot}$ at our most sensitive sky location. We
\end{abstract}

\footnotetext{
${ }_{35}$ NANOGrav Physics Frontiers Center Postdoctoral Fellow.

35 Author order alphabetical by surname.
} 
also determined that there are no SMBHBs with $\mathcal{M}>1.6 \times 10^{9} M_{\odot}$ emitting GWs with $f_{\mathrm{gw}}=2.8-317.8 \mathrm{nHz}$ in the Virgo Cluster. Finally, we compared our strain upper limits to simulated populations of SMBHBs, based on galaxies in the Two Micron All-Sky Survey and merger rates from the Illustris cosmological simulation project, and found that only 34 out of 75,000 realizations of the local universe contained a detectable source.

Key words: gravitational waves - pulsars: general - quasars: supermassive black holes

\section{Introduction}

Pulsar timing arrays (PTAs) seek to detect gravitational waves (GWs) by searching for correlations in the timing observations of a collection of millisecond pulsars (MSPs). The stability of MSPs over long timescales ( decades) makes PTAs ideal detectors for long-wavelength GWs (see Cordes 2013). Currently, there are three PTA experiments in operation: the North American Observatory for Gravitational Waves (NANOGrav; McLaughlin 2013), the European Pulsar Timing Array (EPTA; Desvignes et al. 2016), and the Parkes Pulsar Timing Array (PPTA; Hobbs 2013). Together these groups form the International Pulsar Timing Array (Verbiest et al. 2016). The NANOGrav collaboration has released three data sets based on 5 years of observations (Demorest et al. 2013; hereafter NG5a), 9 years of observations (Arzoumanian et al. 2015; hereafter NG9a), and 11 years of observations (Arzoumanian et al. 2018a; hereafter NG11a).

Potential GW sources in the PTA band include supermassive black hole binaries (SMBHBs; see Sesana et al. 2004; Sesana 2013; Burke-Spolaor et al. 2018), primordial GWs (Grishchuk 2005; Lasky et al. 2016), cosmic strings and superstrings (Damour \& Vilenkin 2001; Ölmez et al. 2010; Blanco-Pillado et al. 2018), and bubble collisions during cosmological phase transitions (Caprini et al. 2010). Historically, analyses have focused on the stochastic gravitational wave background (GWB) formed by the ensemble of a cosmic population of SMBHBs, as models predict that this signal is expected to be detected first (Rosado et al. 2015). In the absence of a detection, constraints have been placed on the GWB, most recently with the NANOGrav $11 \mathrm{yr}$ data set (Arzoumanian et al. 2018b, hereafter NG11b). These limits have been used to narrow the viable parameter space for binary evolution in dynamic galactic environments (e.g., Chen et al. 2017; Taylor et al. 2017; Middleton et al. 2018) and make statements about SMBHB population statistics (e.g., Holgado et al. 2018, Sesana et al. 2018).

PTAs are also sensitive to GWs emitted from nearby individual SMBHBs with periods on the order of months to years, total masses of $\sim 10^{8}-10^{10} M_{\odot}$, and orbital separations of $\sim 10^{-3}-10^{-1} \mathrm{pc}$, depending on the total mass of the binary. SMBHBs that are emitting in the PTA band have nearlyconstant orbital frequencies, hence the GWs from these sources are referred to as "continuous waves" (CWs). However, we do account for the evolution of their orbits over the span of our observations in our analyses. Although there has not yet been a detection of GWs from individual sources with PTAs, they have already been used to place limits on the masses of candidate SMBHBs (e.g., Jenet et al. 2004; Schutz \& Ma 2016). Simulations predict that individual sources will be observed by PTAs within the next 10-20 yr (Rosado et al. 2015; Mingarelli et al. 2017; Kelley et al. 2018).

In this paper, we present the results of searches for GWs from individual circular SMBHBs performed on the NANOGrav $11 \mathrm{yr}$ data set. This is an extension of Arzoumanian et al. (2014) (hereafter NG5b), which performed a similar analysis on the NANOGrav $5 \mathrm{yr}$ data set. Our approach was also inspired by searches performed by the PPTA and EPTA. The first all-sky search for GWs from individual SMBHBs was performed by the PPTA in Yardley et al. (2010), and a later analysis was published in Zhu et al. (2014). The most recent limits on GWs from individual SMBHBs comes from the EPTA (Babak et al. 2016), which performed both Bayesian and frequentist searches for GWs, and placed upper limits as a function of GW frequency and sky location.

The paper is organized as follows. In Section 2, we review the pulsar observations and data reduction techniques used in the creation of the data sets. In Section 3, we describe the GW signal model and noise models used in our search pipelines. We also describe the Bayesian and frequentist methods and software. In Section 4, we present the results of detection searches. As we did not find evidence for GWs in the $11 \mathrm{yr}$ data set, we placed upper limits on the GW strain for $f_{\mathrm{gw}}=2.8-317.8 \mathrm{nHz}$. We also discuss a new analysis technique for identifying spurious signals in PTA data. In Section 5 we present limits on the distances to individual SMBHBs, and limits on the chirp masses of potential SMBHBs in the nearby Virgo Cluster. We also compare our current sensitivity to simulations of SMBHB populations, and estimate the expected number of detectable sources. We conclude in Section 6. Throughout this paper, we use units where $G=c=1$.

\section{The 11 yr Data Set}

We analyzed the NANOGrav $11 \mathrm{yr}$ data set, which was published in NG11a and consisted of times of arrival (TOAs) for 45 pulsars with observations made between 2004 and 2015. Some of these data were previously published as the NANOGrav $5 \mathrm{yr}$ data set in NG5a and the NANOGrav $9 \mathrm{yr}$ data set in NG9a. We briefly review the observations and data reduction techniques here; further details can be found in NG11a.

We made observations using two radio telescopes: the 100m Robert C. Byrd Green Bank Telescope (GBT) of the Green Bank Observatory in Green Bank, West Virginia; and the 305-m William E. Gordon Telescope (Arecibo) of Arecibo Observatory in Arecibo, Puerto Rico. Because Arecibo is more sensitive than GBT, all pulsars that could be observed from Arecibo $\left(0^{\circ}<\delta<39^{\circ}\right)$ were observed with it, while those outside of Arecibo's declination range were observed with GBT. Two pulsars were observed with both telescopes: PSR J1713+0747 and PSR B1937+21. We observed most pulsars once a month. In addition, we started a high-cadence observing campaign in 2013, in which we made weekly observations of two pulsars with GBT (PSR J1713+0747 and PSR J1909-3744) and five pulsars with Arecibo (PSR J00 30+0451，PSR J1640+2224，PSR J1713+0747，PSR J2043 +1711 , and PSR J2317+1439). This high-cadence observing campaign was specifically designed to increase the sensitivity of our PTA to GWs from individual sources (Burt et al. 2011; Christy et al. 2014).

In most cases, we observed pulsars at every epoch with two receivers at different frequencies in order to measure the pulse dispersion due to the interstellar medium (ISM). At GBT, the monthly observations used the $820 \mathrm{MHz}$ and $1.4 \mathrm{GHz}$ 
receivers. The weekly observations used only the $1.4 \mathrm{GHz}$ receiver, which has a wide enough bandwidth to measure the dispersion. At Arecibo, four receivers were used for this project (327 MHz, $430 \mathrm{MHz}, 1.4 \mathrm{GHz}$, and $2.3 \mathrm{GHz}$ ); each pulsar was observed with two different receivers, which were chosen based on the spectral index and timing characteristics of that pulsar. Backend instrumentation was upgraded about midway through our project. Initially, data at Arecibo and GBT were recorded using the ASP and GASP systems, respectively, which had bandwidths of $64 \mathrm{MHz}$. Between 2010 and 2012, we transitioned to the wideband systems PUPPI and GUPPI, which had bandwidths up to $800 \mathrm{MHz}$. Instrumental offsets between the data acquisition systems at each observatory were measured with high precision and were removed from the data to allow for seamless data sets (see NG9a for details).

For each pulsar, the observed TOAs were fit to a timing model that described the pulsar's spin period and spin period derivative, sky location, proper motion, and parallax. The timing model also included terms describing pulse dispersion along the line of sight. Additionally, for those pulsars in binaries the timing model also included five Keplerian parameters that described the binary orbit, and additional post-Keplerian parameters that described relativistic binary effects if they improved the timing fit. In the GW analyses, we used a linearized timing model centered around the best-fit parameter values.

\section{Data Analysis Methods}

PTAs are sensitive to GWs through their effect on the timing residuals. We can write the residuals for each pulsar $\delta t$ as

$$
\delta t=M \epsilon+n_{\text {white }}+n_{\text {red }}+s,
$$

where $M$ is the design matrix, which describes the linearized timing model, $\epsilon$ is a vector of the timing model parameter offsets, $n_{\text {white }}$ is a vector describing white noise, $n_{\text {red }}$ is a vector describing red noise, and $s$ is a vector of the residuals induced by a GW. In this section, we briefly discuss the signal model, likelihood, and methods used in our analyses. These are all similar to those used in NG5b-in the discussion that follows, we emphasize areas in which this analysis differs from previous ones.

\subsection{Signal and Noise Models}

Consider a GW source whose location in equatorial coordinates is given by decl. $\delta$ and R.A. $\alpha$. It is convenient to write the sky position in terms of the polar angle $\theta$ and azimuthal angle $\phi$, which are related to $\delta$ and $\alpha$ by $\theta=\pi /$ $2-\delta$ and $\phi=\alpha$. The emitted GWs can be written in terms of two polarizations:

$$
h_{a b}(t, \hat{\Omega})=e_{a b}^{+}(\hat{\Omega}) h_{+}(t, \hat{\Omega})+e_{a b}^{\times}(\hat{\Omega}) h_{\times}(t, \hat{\Omega}),
$$

where $\hat{\Omega}$ is a unit vector from the GW source to the solar system barycenter (SSB), $h_{+, \times}$are the polarization amplitudes, and $e_{a b}^{+, \times}$are the polarization tensors. The polarization tensors can be written in the SSB frame as (Wahlquist 1987)

$$
\begin{aligned}
& e_{a b}^{+}(\hat{\Omega})=\hat{m}_{a} \hat{m}_{b}-\hat{n}_{a} \hat{n}_{b}, \\
& e_{a b}^{\times}(\hat{\Omega})=\hat{m}_{a} \hat{n}_{b}+\hat{n}_{a} \hat{m}_{b},
\end{aligned}
$$

where

$$
\begin{gathered}
\hat{\Omega}=-\sin \theta \cos \phi \hat{x}-\sin \theta \sin \phi \hat{y}-\cos \theta \hat{z}, \\
\hat{m}=-\sin \phi \hat{x}+\cos \phi \hat{y}, \\
\hat{n}=-\cos \theta \cos \phi \hat{x}-\cos \theta \sin \phi \hat{y}+\sin \theta \hat{z} .
\end{gathered}
$$

The response of a pulsar to the source is described by the antenna pattern functions $F^{+}$and $F^{\times}$(Sesana \& Vecchio 2010; Ellis et al. 2012; Taylor et al. 2016),

$$
\begin{gathered}
F^{+}(\hat{\Omega})=\frac{1}{2} \frac{(\hat{m} \cdot \hat{p})^{2}-(\hat{n} \cdot \hat{p})^{2}}{1+\hat{\Omega} \cdot \hat{p}}, \\
F^{\times}(\hat{\Omega})=\frac{(\hat{m} \cdot \hat{p})(\hat{n} \cdot \hat{p})}{1+\hat{\Omega} \cdot \hat{p}},
\end{gathered}
$$

where $\hat{p}$ is a unit vector pointing from the Earth to the pulsar.

The effect of a GW on a pulsar's residuals can be written as

$$
s(t, \hat{\Omega})=F^{+}(\hat{\Omega}) \Delta s_{+}(t)+F^{\times}(\hat{\Omega}) \Delta s_{\times}(t),
$$

where $\Delta s_{+, \times}$is the difference between the signal induced at the Earth and at the pulsar (the so-called "Earth term" and "pulsar term"),

$$
\Delta s_{+, \times}(t)=s_{+, \times}\left(t_{p}\right)-s_{+, \times}(t),
$$

where $t$ is the time at which the GW passes the SSB and $t_{p}$ is the time at which it passes the pulsar. From geometry, we can relate $t$ and $t_{p}$ by

$$
t_{p}=t-L(1+\hat{\Omega} \cdot \hat{p}),
$$

where $L$ is the distance to the pulsar.

For a circular binary, at zeroth post-Newtonian (0-PN) order, $s_{+, \times}$is given by (Wahlquist 1987; Corbin \& Cornish 2010; Lee et al. 2011)

$$
\begin{aligned}
s_{+}(t)= & \frac{\mathcal{M}^{5 / 3}}{d_{L} \omega(t)^{1 / 3}}\left[-\sin 2 \Phi(t)\left(1+\cos ^{2} i\right) \cos 2 \psi\right. \\
& -2 \cos 2 \Phi(t) \cos i \sin 2 \psi], \\
s_{\times}(t)= & \frac{\mathcal{M}^{5 / 3}}{d_{L} \omega(t)^{1 / 3}}\left[-\sin 2 \Phi(t)\left(1+\cos ^{2} i\right) \sin 2 \psi\right. \\
& +2 \cos 2 \Phi(t) \cos i \cos 2 \psi],
\end{aligned}
$$

where $i$ is the inclination angle of the SMBHB, $\psi$ is the GW polarization angle, $d_{L}$ is the luminosity distance to the source, and $\mathcal{M} \equiv\left(m_{1} m_{2}\right)^{3 / 5} /\left(m_{1}+m_{2}\right)^{1 / 5}$ is a combination of the black hole masses $m_{1}$ and $m_{2}$, which is called the "chirp mass." Note that the variables $\mathcal{M}$ and $\omega$ are the observed redshifted values, which are related to the rest-frame values $\mathcal{M}_{r}$ and $\omega_{r}$ according to

$$
\begin{gathered}
\mathcal{M}_{r}=\frac{\mathcal{M}}{1+z}, \\
\omega_{r}=\omega(1+z) .
\end{gathered}
$$

Currently PTAs are only sensitive to sources in the local universe for which $(1+z) \approx 1$.

For a circular binary, the orbital angular frequency is related to the GW frequency by $\omega_{0}=\pi f_{\mathrm{gw}}$, where $\omega_{0}=\omega\left(t_{0}\right)$. For our search, we defined the reference time $t_{0}$ as 2015 December 31 (MJD 57387), which corresponded to the last day data were taken for the $11 \mathrm{yr}$ data set. The orbital phase and frequency of 
the SMBHB are given by (NG5b)

$$
\begin{gathered}
\Phi(t)=\Phi_{0}+\frac{1}{32} \mathcal{M}^{-5 / 3}\left[\omega_{0}^{-5 / 3}-\omega(t)^{-5 / 3}\right], \\
\omega(t)=\omega_{0}\left(1-\frac{256}{5} \mathcal{M}^{5 / 3} \omega_{0}^{8 / 3} t\right)^{-3 / 8},
\end{gathered}
$$

where $\Phi_{0}$ and $\omega_{0}$ are the initial orbital phase and frequency, respectively. Unlike in NG5b, we used the full expression for $\omega(t)$ in our signal model rather than treating the GW frequency at the Earth as a constant, as high-chirp-mass binaries will evolve significantly over the timescale of our observations.

Our noise model for individual pulsars included both white noise and red noise. We used the same white noise model as NG5b, which has three parameters: EFAC, EQUAD, and ECORR. The EFAC parameter scales the TOA uncertainties, and the EQUAD parameter adds white noise in quadrature. The ECORR parameter describes additional white noise added in quadrature that is correlated within the same observing epoch, such as pulse jitter (Dolch et al. 2014; Lam et al. 2017). We used the improved implementation of ECORR described in NG11b. To model the red noise, we divided the noise spectrum into 30 bins spaced linearly between $f=1 / T_{\text {obs }}$ and $f=30 / T_{\text {obs }}$, where $T_{\text {obs }}$ is the total observation time for a particular pulsar, ${ }^{36}$ and then fit the power spectral density to a power-law model,

$$
P(f)=A_{\text {red }}^{2}\left(\frac{f}{f_{\mathrm{yr}}}\right)^{-\gamma},
$$

where $f_{\mathrm{yr}} \equiv 1 /(1 \mathrm{yr}), A_{\text {red }}$ is the amplitude, and $\gamma$ is the spectral index. There are many possible sources of red noise in pulsar timing residuals, including spin noise, variations in pulse shape, pulsar mode changes, and errors in modeling pulse dispersion from the ISM (Cordes 2013; Jones et al. 2017; Lam et al. 2017). We model time variations in the ISM through DMX parameters, which measure the dispersion at almost every observing epoch (NG9a, Lam et al. 2016).

\subsection{Bayesian Methods and Software}

We used Bayesian inference to determine posterior distributions of GW parameters from our data. The procedure followed closely that of NG5b, with the addition of the BAYESEPHEM model for the uncertainty in the SSB introduced in NG11b. Pulsar timing uses a solar system ephemeris (SSE) to transform from individual observatories' reference frames to an inertial reference frame centered at the SSB. We used DE436 (Folkner \& Park 2016) to perform this transformation, plus the BAYESEPHEM model. Uncertainty in the SSE has a significant impact on the computation of GW upper limits from PTA data. The BAYESEPHEM model mitigates this by marginalizing over perturbations in the outer planets' masses and Jupiter's orbit. This approach removes systematic uncertainty in the position of the SSB by introducing statistical uncertainty through the addition of new parameters. Another approach to differentiating between GW signals and uncertainty in the SSE, which we do not explore in this paper, is to exploit the fact that the two

\footnotetext{
36 Van Haasteren \& Vallisneri (2015) introduced a better method for choosing the frequency basis for red noise, which reduces the computational cost However, in this work we chose a linear frequency basis to make it easier to compare these results with the results in NG5b.
}

have different spatial correlations (Tiburzi et al. 2016). A detailed analysis of how errors in the SSE effect PTAs can be found in Caballero et al. (2018).

We used the same likelihood as in NG5b. We implemented the likelihood and priors and performed the searches using NANOGrav's new software package enterprise. ${ }^{37} \mathrm{We}$ confirmed the accuracy of this package by also performing some searches using the software package PAL $2,{ }^{38}$ which has been used for previous NANOGrav GW searches. Both packages used the Markov Chain Monte Carlo (MCMC) sampler PTMCMCSampler ${ }^{39}$ to explore the parameter space.

For detection and upper-limit runs, we described the Earthterm contribution to the GW signal by eight parameters:

$$
\lambda_{0}=\left\{\theta, \phi, \Phi_{0}, \psi, i, \mathcal{M}, f_{\mathrm{gw}}, h_{0}\right\},
$$

where the characteristic strain $h_{0}$ is related to $\mathcal{M}, f_{\mathrm{gw}}$, and $d_{L}$ according to

$$
h_{0}=\frac{2 \mathcal{M}^{5 / 3}\left(\pi f_{\mathrm{gw}}\right)^{2 / 3}}{d_{L}} .
$$

We used log-uniform priors on $h_{0}$ for detection analyses, and a uniform prior on $h_{0}$ to compute upper limits on the strain. For both types of analyses, we searched over $\log _{10} h_{0} \in[-18,-11]$.

We used isotropic priors on the sky position of the source $(\theta, \phi)$, source inclination angle $i, \mathrm{GW}$ polarization angle $\psi$, and GW phase $\Phi_{0}$. We searched over $\log _{10} \mathcal{M}$ with a uniform prior $\log _{10}\left(\mathcal{M} / M_{\odot}\right) \in[7,10]$. For high $f_{\mathrm{gw}}$, we truncated the prior on $\log _{10} \mathcal{M}$ to account for the fact that high-chirp-mass systems will have merged before emitting high-frequency GWs. Assuming binaries merge when the orbital frequency is equal to the innermost stable circular orbit frequency, $\mathcal{M}$ must satisfy

$$
\mathcal{M} \leqslant \frac{1}{6^{3 / 2} \pi f_{\mathrm{gw}}}\left[\frac{q}{(1+q)^{2}}\right]^{3 / 5},
$$

where $q$ is the mass ratio. For our analyses, we used the chirpmass cutoff with $q=1$. This change to the prior on $\mathcal{M}$ only affected $f_{\mathrm{gw}} \geqslant 191.3 \mathrm{nHz}$.

We performed searches at fixed values of $f_{\mathrm{gw}}$. The minimum GW frequency was set by the total observation time, $f_{\mathrm{gw}}=1 /(11.4 \mathrm{yr})=2.8 \mathrm{nHz}$. The maximum GW frequency was set by the observing cadence. Because of the high-cadence observing campaign, the $11 \mathrm{yr}$ data set can detect GWs with frequencies up to $826.7 \mathrm{nHz}$; however, the data are not very sensitive at high frequencies. Also, we do not expect to find any SMBHBs with orbital periods of weeks because highchirp-mass systems would have already merged before emitting at those frequencies, and low-chirp-mass systems would be evolving through the PTA band very quickly at that point. Therefore, we only searched for GWs with frequencies up to $317.8 \mathrm{nHz}$, which corresponded to the high-frequency-cutoff adopted in NG5b.

The pulsar-term contributions to the GW signal used the pulsar distances to compute the light-travel-time between when the GW passed the pulsars and when it passed the SSB (see Equation (12)). We used a Gaussian prior on the distances with the measured mean and uncertainty from Verbiest et al. (2012);

\footnotetext{
37 https://github.com/nanograv/enterprise

38 https://github.com/jellis18/PAL2

39 https://github.com/jellis18/PTMCMCSampler
} 
for the pulsars not included in that paper, we used a mean of $1 \mathrm{kpc}$ and error of $20 \%$. The use of approximate distances for some pulsars did not seem to affect our results-we found that the pulsar distance posteriors were identical to the priors, indicating that the data are insensitive to the pulsar distances. Furthermore, we only used approximate pulsar distances for pulsars that had only been observed for a few years, and therefore did not contribute significantly to the GW sensitivity. The phase at the pulsar can be written as

$$
\Phi(t)=\Phi_{0}+\Phi_{p}+\frac{1}{32} \mathcal{M}^{-5 / 3}\left[\omega\left(t_{p, 0}\right)^{-5 / 3}-\omega\left(t_{p}\right)^{-5 / 3}\right],
$$

where $\Phi_{p}$ is the phase difference between the Earth and the pulsar. The pulsar phase parameters $\Phi_{p}$ can be computed from the pulsar distances and chirp mass as

$$
\Phi_{p}=\frac{1}{32} \mathcal{M}^{-5 / 3}\left[\omega_{0}^{-5 / 3}-\omega\left(t_{p, 0}\right)^{-5 / 3}\right] ;
$$

however, in most cases the pulsar distance uncertainties $(\Delta L \sim 10-100 \mathrm{pc})$ are significantly greater than the GW wavelengths $\left(\lambda_{\mathrm{gw}} \sim 0.1-10 \mathrm{pc}\right)$, so the phase differences between the Earth terms and pulsar terms are effectively random. Therefore, following the approach of Corbin \& Cornish (2010), we treated $\Phi_{p}$ as an independent parameter with a uniform prior $\Phi_{p} \in[0,2 \pi]$.

We fixed the white noise parameters to their best-fit values, as determined from noise analyses performed on individual pulsars. In the GW analyses, we simultaneously searched over the individual pulsars' red noise using a power-law model with uniform priors on $\log _{10} A_{\text {red }} \in[-20,-11]$ and $\gamma \in[0,7]$. In order to burn-in the red noise and BAYESEPHEM parameters efficiently, we introduced jump proposals that drew proposed samples from empirical distributions based on the posteriors from an initial Bayesian analysis with only the pulsars' red noise and BAYESEPHEM (i.e., excluding a GW signal). For more details, see the Appendix.

We computed Bayes factors for the presence of a GW signal using the Savage-Dickey formula (Dickey 1971),

$$
\mathcal{B}_{10} \equiv \frac{\text { evidence }\left[\mathcal{H}_{1}\right]}{\text { evidence }\left[\mathcal{H}_{0}\right]}=\frac{p\left(h_{0}=0 \mid \mathcal{H}_{1}\right)}{p\left(h_{0}=0 \mid \mathcal{D}, \mathcal{H}_{1}\right)},
$$

where $\mathcal{H}_{1}$ is the model with a GW signal plus individual pulsar red noise, $\mathcal{H}_{0}$ is the model with only individual pulsar red noise, $p\left(h_{0}=0 \mid \mathcal{H}_{1}\right)$ is the prior volume at $h_{0}=0$, and $p\left(h_{0}=0 \mid \mathcal{D}, \mathcal{H}_{1}\right)$ is the posterior volume at $h_{0}=0$. We were able to use the Savage-Dickey formula because $\mathcal{H}_{1}$ and $\mathcal{H}_{0}$ are nested models, i.e., $\mathcal{H}_{0}$ is $\mathcal{H}_{1}: h_{0}=0$. We approximated $p\left(h_{0}=0 \mid \mathcal{D}, \mathcal{H}_{1}\right)$ as the fraction of quasi-independent samples in the lowest-amplitude bin of a histogram of $h_{0}$. We found the quasi-independent samples by thinning the chain by the autocorrelation chain length, which is a measure of how far apart two samples in the chain must be in order to be statistically independent. We computed the error in the Bayes factor as

$$
\sigma=\frac{\mathcal{B}_{10}}{\sqrt{n}}
$$

where $n$ is the number of samples in the lowest-amplitude bin.
For upper limits, following the approach of NG11b, we computed the standard error as

$$
\sigma=\frac{\sqrt{x(1-x) / N_{s}}}{p\left(h_{0}=h_{0}^{95 \%} \mid \mathcal{D}\right)},
$$

where $x=0.95$ and $N_{s}$ is the number of effective samples in the chain. This definition of $\sigma$ is the error in the computed $95 \%$ upper limit due to using a finite number of samples. We estimated the number of effective samples by dividing the total number of samples by the autocorrelation chain length, which is a measure of how far apart two samples in the chain must be in order to be statistically independent.

\section{3. $\mathcal{F}_{p}$-statistic}

As in NG5b, we also performed a frequentist analysis with the $\mathcal{F}_{p}$-statistic, which we computed using the software package enterprise. The $\mathcal{F}_{p}$-statistic is an incoherent detection statistic that is derived by maximizing the $\log$ of the likelihood ratio (Ellis et al. 2012). Essentially, it is the weighted sum of the power spectrum of the residuals, summed over all pulsars. This statistic assumes the SMBHB's orbital frequency is not evolving significantly over the timescale of our observations. In the absence of a signal, $2 \mathcal{F}_{p}$ follows a chisquared distribution with $2 N_{p}$ degrees of freedom, where $N_{p}$ is the number of pulsars. The corresponding false-alarm-probability (FAP) is

$$
P_{F}\left(\mathcal{F}_{p, 0}\right)=\exp \left(-\mathcal{F}_{p, 0}\right) \sum_{k=0}^{N_{p}-1} \frac{\mathcal{F}_{p, 0}^{k}}{k !} .
$$

In performing $\mathrm{GW}$ searches over our entire frequency range, we compute the $\mathcal{F}_{p}$ statistic $N_{f}$ times, where $N_{f}$ is the number of independent frequencies, i.e., the number of frequencies separated by $1 / T_{\mathrm{obs}}=2.7 \mathrm{nHz}$. The FAP for the entire search is

$$
P_{F}^{T}\left(\mathcal{F}_{p, 0}\right)=1-\left[1-P_{F}\left(\mathcal{F}_{p, 0}\right)\right]^{N_{f}} .
$$

For the analysis in this paper, $N_{f}=115$.

\section{Results}

In this section we report the results of both detection and upper limit analyses of the NANOGrav $11 \mathrm{yr}$ data set for GWs from individual circular SMBHBs. We used the data to place upper limits as a function of frequency and sky location, and to compare upper limits from the $11 \mathrm{yr}$ data set to those from the 5 and $9 \mathrm{yr}$ data sets. We also discuss a new Bayesian technique to determine how much each pulsar in a PTA contributes to a common signal in order to diagnose spurious signals. Following the approach of NG11b, our analyses of the $11 \mathrm{yr}$ data set only used the 34 pulsars that had been observed for at least three years. Our analyses of the 5 and $9 \mathrm{yr}$ data sets used the same subset of pulsars that were used in the corresponding analyses for the GWB (NG5a, Arzoumanian et al. 2016), which included 17 and 18 pulsars, respectively.

\subsection{Detection Analyses}

We performed detection searches for GWs from individual circular SMBHBs on the $11 \mathrm{yr}$ data set. Figure 1 shows the Bayes factors for each frequency, marginalized over the sky location. We did not find strong evidence for GWs in the $11 \mathrm{yr}$ 


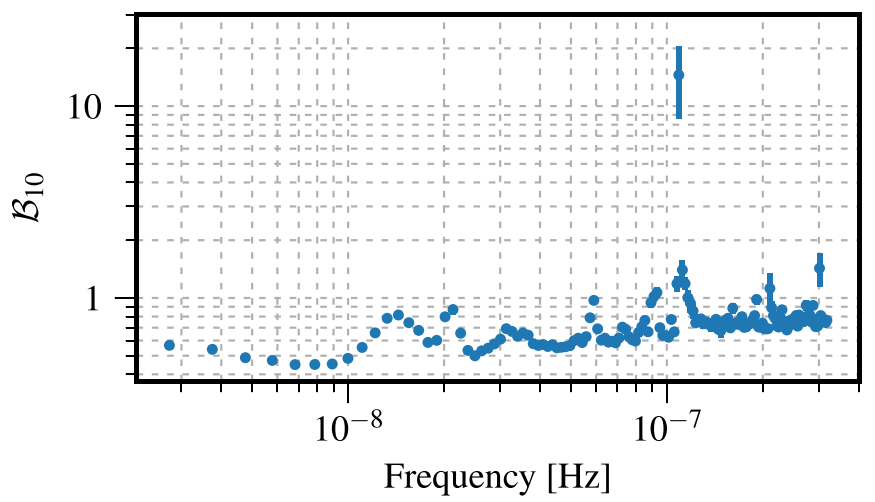

Figure 1. Bayes factors for a GW signal from an individual circular SMBHB as a function of GW frequency in the NANOGrav $11 \mathrm{yr}$ data set. We found no strong evidence for GWs in our data. The highest Bayes factor was at $f_{\mathrm{gw}}=109 \mathrm{nHz}$, for which $\mathcal{B}_{10}=15(6)$. For all other frequencies searched, the Bayes factors were close to 1 .

data set. The largest Bayes factor was at $f_{\mathrm{gw}}=109 \mathrm{nHz}$, for which $\mathcal{B}_{10}=15(6)$. For all other frequencies, the Bayes factors were between $\mathcal{B}_{10}=0.449(4)$ and $\mathcal{B}_{10}=1.4(3)$, indicating no evidence of GWs in the data.

We also used the $\mathcal{F}_{p}$-statistic to determine the significance of a GW signal. Figure 2 shows the $\mathcal{F}_{p}$-statistic as a function of $f_{\mathrm{gw}}$, and the corresponding FAP as computed from Equation (28). There are no frequencies for which the FAP lies below our detection threshold of $10^{-4}$. At the GW frequency that maximizes $\mathcal{F}_{p}$, the total FAP for the search as computed from Equation (29) is $P_{F}^{T}\left(\mathcal{F}_{p, 0}\right)=0.543$. Thus, we concluded that the frequentist analyses also found that the $11 \mathrm{yr}$ data set does not contain significant evidence for GWs.

Although the detection search at $f_{\mathrm{gw}}=109 \mathrm{nHz}$ found a higher Bayes factor than any of the other values of $f_{\mathrm{gw}}$, we emphasize that the Bayes factor is not high enough to claim a detection. A Bayes factor of 15 means 15:1 odds for the presence of a GW signal; similarly, at this frequency the $\mathcal{F}_{p}$-statistic corresponds to $P_{F}\left(\mathcal{F}_{p, 0}\right)=0.235$, or a signal-tonoise ratio $(\mathrm{S} / \mathrm{N})$ of 1.2. Neither of these metrics supports the claim that the data show evidence of GWs. Furthermore, as we discuss in more detail in Section 4.3, we determined that most of the evidence for this signal was in the residuals of a single pulsar, $\mathrm{J} 1713+0747$, whereas a true GW signal that is this strong should be seen in many pulsars.

\subsection{Upper Limit Analyses}

As we did not find strong evidence for GWs from individual circular SMBHBs in the $11 \mathrm{yr}$ data set, we placed upper limits on the GW strain. Figure 3 shows the sky-averaged 95\% upper limit on the GW strain amplitude. At the most sensitive frequency of $8 \mathrm{nHz}$, we placed a $95 \%$ upper limit on the strain of approximately $h_{0}<7.3(3) \times 10^{-15}$. We also show the strain upper limits from the 5 and $9 \mathrm{yr}$ data sets for comparison. There was an improvement of about a factor of two between the $5 \mathrm{yr}$ and $9 \mathrm{yr}$ data sets, and more than a factor of two between the $9 \mathrm{yr}$ and $11 \mathrm{yr}$ data sets. Our upper limit based on the $11 \mathrm{yr}$ data set was about 1.4 times lower than that of $h_{0}<10^{-14}$ set by the EPTA based on observations of 6 pulsars observed for up to $17.7 \mathrm{yr}$ (Babak et al. 2016; Desvignes et al. 2016). However, a direct comparison between the EPTA results and the results in this paper is complicated by the fact that the analysis in Babak et al. (2016) varied both the white and red

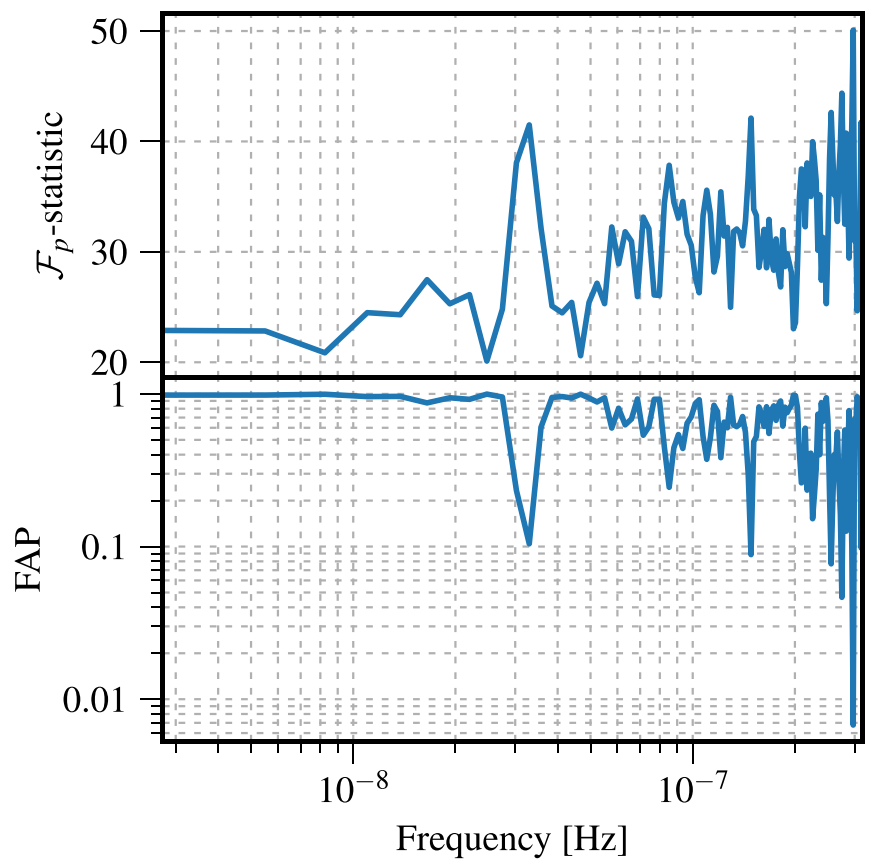

Figure 2. $\mathcal{F}_{p}$-statistic (top panel) and the corresponding FAP (bottom panel) for $f_{\mathrm{gw}}=2.8-317.8 \mathrm{nHz}$. There were no frequencies for which the FAP was below our detection threshold of $10^{-4}$; therefore, we concluded there was no evidence for GWs.

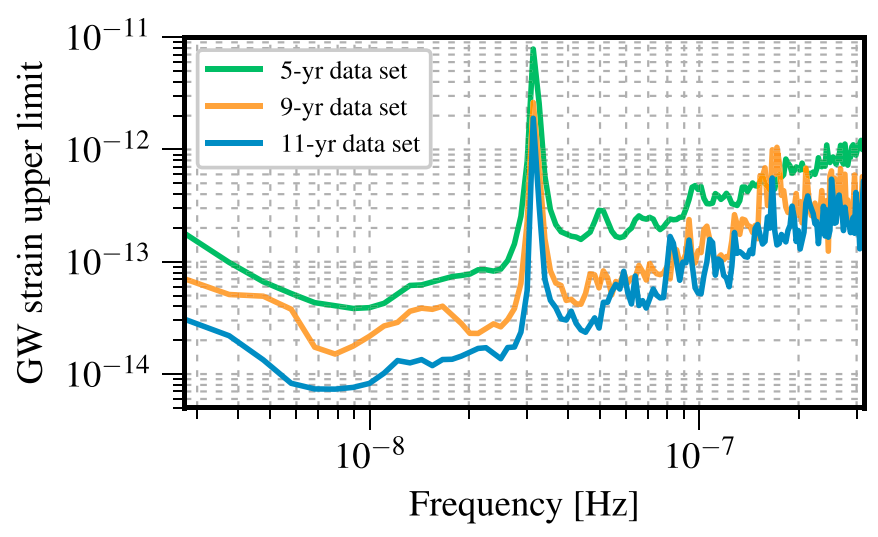

Figure 3. Sky-averaged $95 \%$ upper limit on the GW strain amplitude as a function of GW frequency from the NANOGrav $5 \mathrm{yr}$ data set (green), $9 \mathrm{yr}$ data set (orange), and $11 \mathrm{yr}$ data set (blue). These analyses used BAYESEPHEM to parameterize uncertainty in the SSB. The data were most sensitive at $f_{\mathrm{gw}}=8 \mathrm{nHz}$, with a strain upper limit of approximately $h_{0}<1.51$ (7) $\times 10^{-14}$ from the $9 \mathrm{yr}$ data set, and $h_{0}<7.3(3) \times 10^{-15}$ from the $11 \mathrm{yr}$ data set.

noise, whereas our analysis varied only the red noise and fixed the white noise. Our upper limit is also about a factor of 2 lower than that of $h_{0}<1.7 \times 10^{-14}$ set by the PPTA using their Data Release 1 (Manchester et al. 2013; Zhu et al. 2014)

We note that there is an increase in the strain upper limit from the $9 \mathrm{yr}$ data set at around $f_{\mathrm{gw}}=15 \mathrm{nHz}$; however, there is not a significant Bayes factor at this frequency $\left(\mathcal{B}_{10}=1.4(1)\right)$. Furthermore, this "bump" in the spectrum is not present in the $11 \mathrm{yr}$ data set. If it were caused by a $\mathrm{GW}$, the significance should have increased in the $11 \mathrm{yr}$ data set. As discussed in more detail in Section 4.3, this increase in the strain upper limit is due to an unmodeled signal in a single pulsar, PSR J0613-0200. 


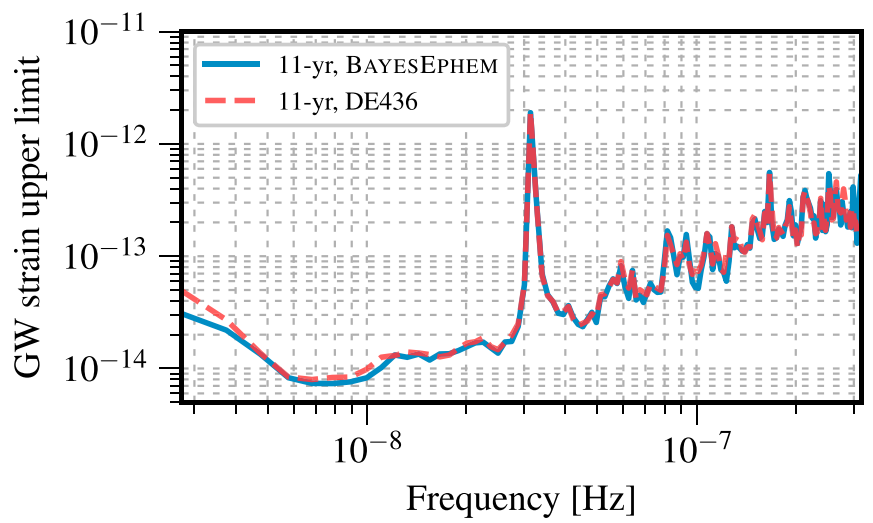

Figure 4. Sky-averaged $95 \%$ upper limit on the GW strain amplitude from a circular SMBHB as a function of GW frequency, with and without BAYESEPHEM (solid blue curve and dashed red curve, respectively). At the lowest frequencies $\left(f_{\mathrm{gw}} \lesssim 4 \mathrm{nHz}\right)$, the analysis with BAYESEPHEM was more sensitive than the analysis without it, but there was no difference in sensitivity at higher frequencies.

In Figure 4, we compare the sky-averaged strain upper limits computed with and without BAYESEPHEM, which allows for uncertainties in the SSE. Including BAYESEPHEM in our model resulted in a lower strain upper limit for $f_{\mathrm{gw}}<4 \mathrm{nHz}$, but did not affect the strain upper limit at higher frequencies. This was expected because BAYESEPHEM primarily augments the orbit of Jupiter, which has an orbital period of $12 \mathrm{yr}$.

Our sensitivity to individual sources varied significantly with the angular position of the source due to having a finite number of pulsars distributed unevenly across the sky. Figure 5 shows the $95 \%$ upper limit on the GW strain for $f_{\mathrm{gw}}=8 \mathrm{nHz}$ as a function of sky position, plotted in equatorial coordinates. The upper limit varies from $h_{0}<2.0(1) \times 10^{-15}$ at the most sensitive sky location to $h_{0}<1.34(4) \times 10^{-14}$ at the least sensitive sky location.

\section{3. "Dropout” Analyses}

Our searches of the NANOGrav $9 \mathrm{yr}$ and $11 \mathrm{yr}$ data sets found two low-S/N signals. In order to identify their sources, we introduced a new type of analysis that used "dropout" parameters to determine how much each individual pulsar contributed to these signals. In a dropout analysis, the model for a pulsar's residuals [Equation (1)] is modified so that the GW signal can be turned on or off in each individual pulsar:

$$
\delta t=M \epsilon+n_{\text {white }}+n_{\text {red }}+\kappa s,
$$

where $\kappa \in\{0,1\}$. The GW parameters were held fixed at the values that maximized the likelihood of a standard GW search, and dropout parameters $k_{a}$ were introduced into the signal model, which were drawn from a uniform prior between 0 and 1. These parameters determined whether the signal was turned on or off in a particular pulsar:

$$
\kappa_{a}=\left\{\begin{array}{ll}
0 & k_{a}<k_{\text {threshold }} \\
1 & k_{a} \geqslant k_{\text {threshold }}
\end{array},\right.
$$

where $k_{\text {threshold }}$ sets the prior on whether the signal should be included in a pulsar. For the analyses in this paper, we used $k_{\text {threshold }}=1 / 2$, meaning that the prior assumed it was equally likely that the GW be turned on or off. At each iteration of the MCMC, the astrophysical properties of the GW were fixed, and the only thing that varied was which pulsars' residuals
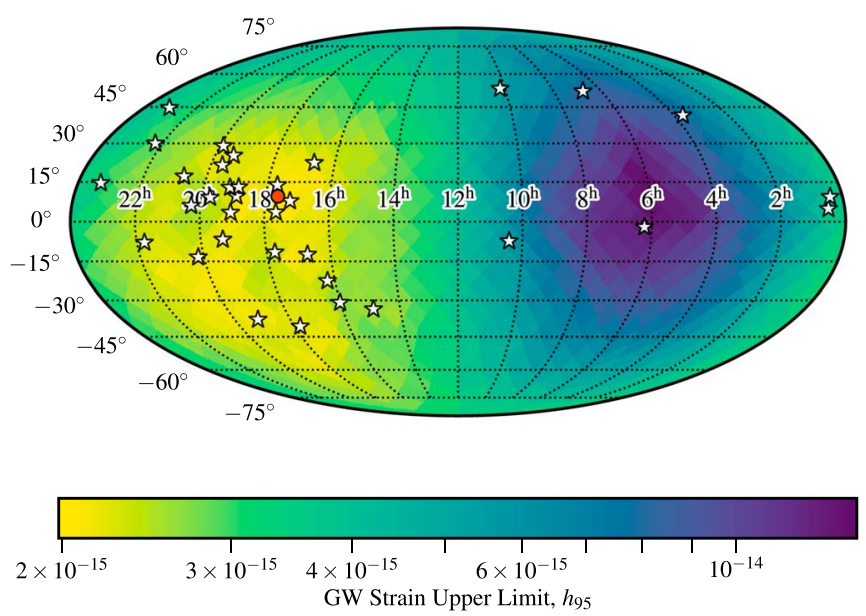

Figure 5. The $95 \%$ upper limit on the GW strain amplitude from a circular SMBHB with $f_{\mathrm{gw}}=8 \mathrm{nHz}$ as a function of sky position from an analysis of the $11 \mathrm{yr}$ data set, plotted in equatorial coordinates using the Mollweide projection. We used the DE436 ephemeris model with BAYESEPHEM to model uncertainty in the SSB. The positions of pulsars in our array are indicated by stars, and the most sensitive sky location is indicated by a red circle. The $95 \%$ upper limit ranged from $2.0(1) \times 10^{-15}$ at our most sensitive sky location to $1.34(4) \times 10^{-14}$ at our least sensitive sky location.

contained the GW signal. The posteriors of the dropout parameters indicated how much support there was for the GW in each pulsar.

The dropout method tests the robustness of the correlations in the signal by determining whether evidence for the signal comes from correlations between multiple pulsars, or if it only originates from a single pulsar. It is similar to the dropout technique in neural networks, where units are randomly dropped during training in order to strengthen the network (Srivastava et al. 2014). This method is also similar to jackknife resampling (Efron \& Stein 1981); however, in jackknifing, samples are removed in order to estimate the bias in parameter estimation, whereas in dropout analyses the parameter values are held fixed, and the dropout parameters indicate how much each pulsar is biasing the parameter estimation. An upcoming paper will further describe and develop this method (S. J. Vigeland et al. 2019, in preparation)

We performed two dropout analyses. The first was on the $9 \mathrm{yr}$ data set at $f_{\mathrm{gw}}=15 \mathrm{nHz}$. The analysis of the $9 \mathrm{yr}$ data set found an increase in the $95 \%$ strain upper limit at $f_{\mathrm{gw}}=15 \mathrm{nHz}$ compared to the upper limits at neighboring frequencies. Furthermore, as shown in Figure 6, we found that the strain upper limit decreased significantly when PSR J0613 -0200 was removed from the $9 \mathrm{yr}$ data set. However, there was very little difference in the Bayes factor: $\mathcal{B}_{10}=1.4(1)$ with all pulsars, and $\mathcal{B}_{10}=1.11(4)$ excluding PSR J0613-0200. Figure 7 shows the results of a dropout analysis. We fixed the GW signal parameters to the best-fit values from a detection analysis including all pulsars, and only allowed the dropout parameters to vary. We set $k_{\text {threshold }}=1 / 2$, so that there was an equal prior probability of the signal being included or excluded in the model for each pulsar's residuals. PSR J0613-0200 had the largest Bayes factor while all other pulsars had Bayes factors of order 1, from which we concluded that the increase in the strain upper limit at $f_{\mathrm{gw}}=15 \mathrm{nHz}$ was caused by an unmodeled non-GW signal in PSR J0613-0200. We have applied advanced noise modeling techniques to this pulsar, using more complex models for the red noise, and modeling 


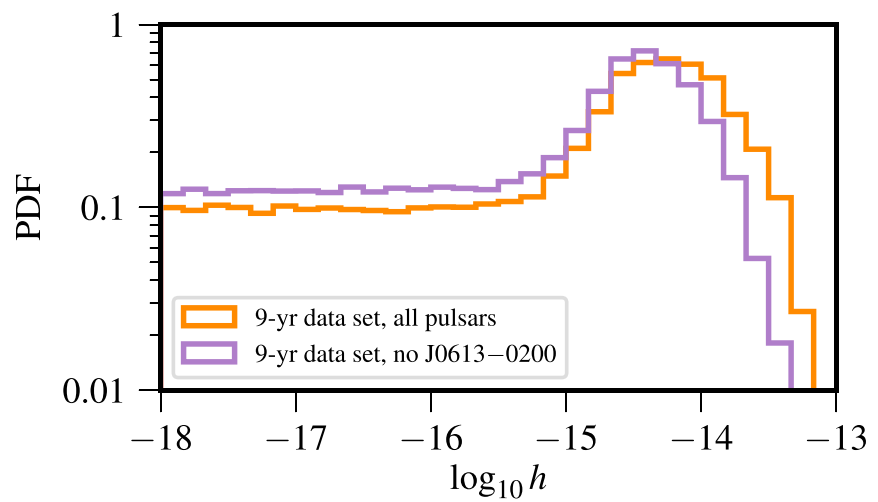

Figure 6. Comparison between a search at $f_{\mathrm{gw}}=15 \mathrm{nHz}$ performed on the $9 \mathrm{yr}$ data set with all pulsars (orange) and excluding PSR J0613-0200 (purple). There was very little difference between the Bayes factors $\left(\mathcal{B}_{10}=1.4(1)\right.$ with all pulsars, and $\mathcal{B}_{10}=1.11$ (4) excluding PSR J0613 -0200 ), but there was a significant difference in the $95 \%$ strain upper limit. We found an upper limit of 4.1(2) $\times 10^{-14}$ with all pulsars, compared with 3.2 (3) $\times 10^{-14}$ without PSR J0613-0200.

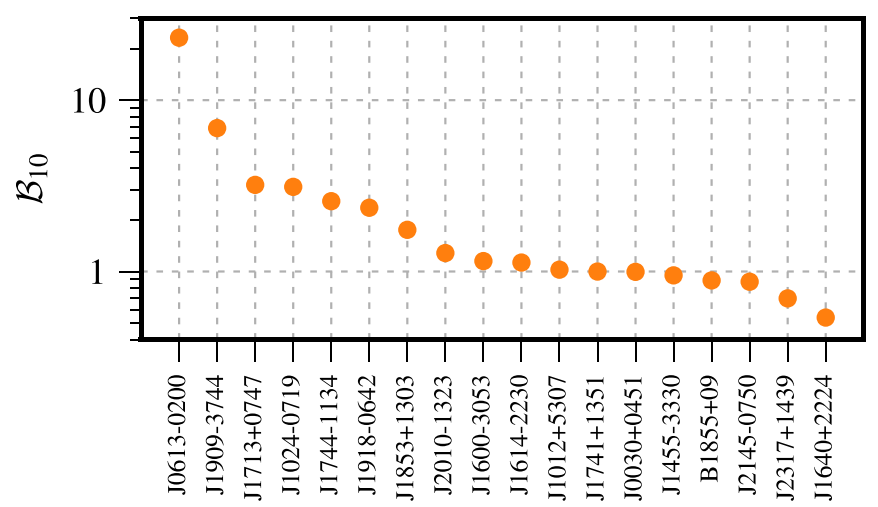

Figure 7. Bayes factors for the presence of a GW signal in each pulsar's residuals, from an analysis of the $9 \mathrm{yr}$ data set with $f_{\mathrm{gw}}=15 \mathrm{nHz}$. The GW parameters are fixed to the maximum-likelihood values, as determined from a detection analysis. PSR J0613-0200 had the largest Bayes factor for the signal, with $\mathcal{B}_{10}=23.2(5)$, indicating that PSR J0613-0200 was the primary source of this signal.

time-dependent variations in the dispersion as a Gaussian process rather than with DMX. These results will be discussed in an upcoming paper.

We also performed a dropout analysis on the $11 \mathrm{yr}$ data set at $f_{\mathrm{gw}}=109 \mathrm{nHz}$, for which the detection searches had found $\mathcal{B}_{10}=15(6)$. Figure 8 shows the Bayes factors for each pulsar's dropout parameter. We found that PSR J1713+0747 had the strongest Bayes factor for including a GW signal at this frequency, with $\mathcal{B}_{10}=96.2(1)$, indicating that most of the evidence for this signal comes from the residuals of PSR J1713 +0747 . We did not perform an analysis removing PSR J1713 +0747 because it is one of the most sensitive pulsars in the NANOGrav PTA, and removing it always decreases our sensitivity to GWs. Because J1713+0747 significantly contributes to every $\mathrm{GW}$ analysis, it is unsurprising that noise in this pulsar can be confused for a GW. A noise analysis of $\mathrm{J} 1713+0747$ is underway using the advanced noise modeling techniques that were also applied to J0613-0200, and the results will be discussed in an upcoming paper. Future CW analyses of PTA data will be able to definitively determine the source of this signal with additional timing data and the

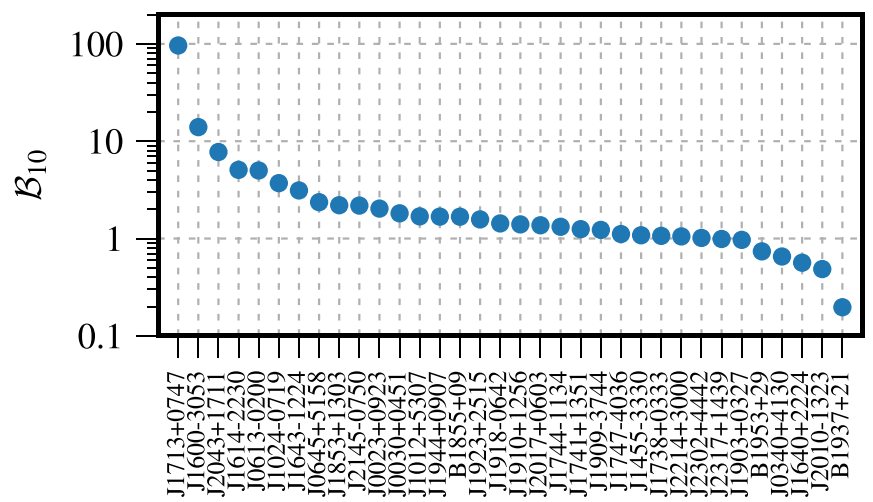

Figure 8. Bayes factors for the presence of a GW signal in each pulsar's residuals, from an analysis of the $11 \mathrm{yr}$ data set with $f_{\mathrm{gw}}=109 \mathrm{nHz}$. The GW parameters were fixed to the maximum-likelihood values, as determined from a detection analysis. We concluded that this signal was primarily coming from PSR J1713+0747, for which $\mathcal{B}_{10}=96.2(1)$.
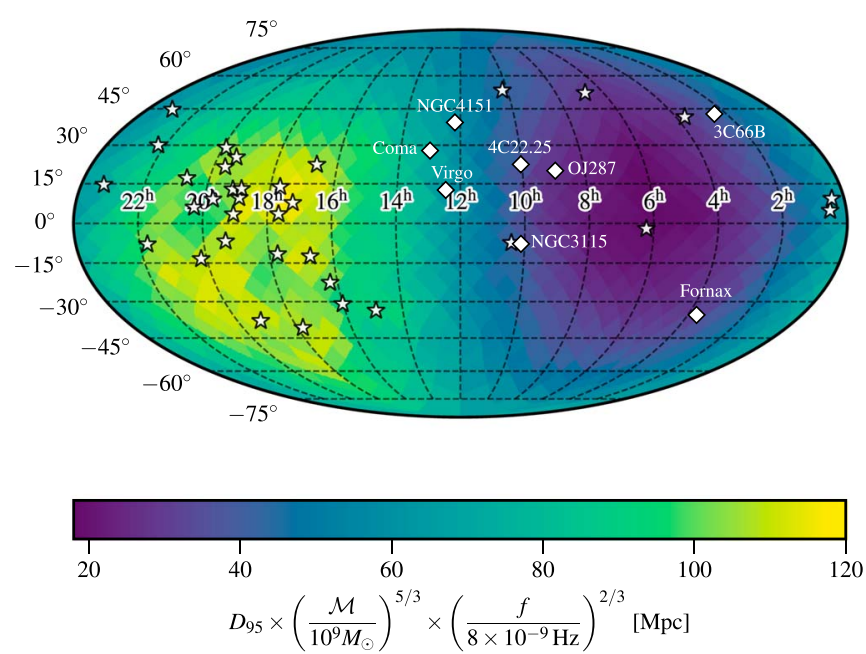

Figure 9. The $95 \%$ lower limit on the distance to individual SMBHBs with $\mathcal{M}=10^{9} M_{\odot}$ and $f_{\mathrm{gw}}=8 \mathrm{nHz}$ as a function of sky position based on an analysis of the $11 \mathrm{yr}$ data set, plotted in equatorial coordinates using the Mollweide projection. The stars indicate the positions of pulsars in our array, and the diamonds indicate the positions of known SMBHB candidates or galaxy clusters that may contain SMBHBs. At our most sensitive sky location, we place a limit of $d_{L}>120 \mathrm{Mpc}$ for SMBHBs with $\mathcal{M}=10^{9} M_{\odot}$, and $d_{L}>5.5 \mathrm{Gpc}$ for SMBHBs with $\mathcal{M}=10^{10} M_{\odot}$.

incorporation of advanced noise modeling techniques into the data analysis pipeline.

\section{Limits on Astrophysical Properties of Nearby SMBHBs}

In this section, we discuss what we can infer about the astrophysical properties of nearby SMBHBs from our limits on the GW strain. We used the $95 \%$ upper limits on the GW strain to place $95 \%$ lower limits on the distance to SMBHBs using Equation (21) for a given chirp mass. Figure 9 shows the 95\% lower limit on the distances to individual SMBHBs as a function of sky position, plotted in equatorial coordinates, for sources with $\mathcal{M}=10^{9} M_{\odot}$ and $f_{\mathrm{gw}}=8 \mathrm{nHz}$. The limits on the luminosity distance varied by a factor of 7 between the most sensitive and least sensitive sky locations. At the most sensitive sky location, we found $d_{L}>120 \mathrm{Mpc}$ for SMBHBs with $\mathcal{M}=10^{9} M_{\odot}$ and $d_{L}>5.5 \mathrm{Gpc}$ for SMBHBs with $\mathcal{M}=10^{10} M_{\odot}$. 


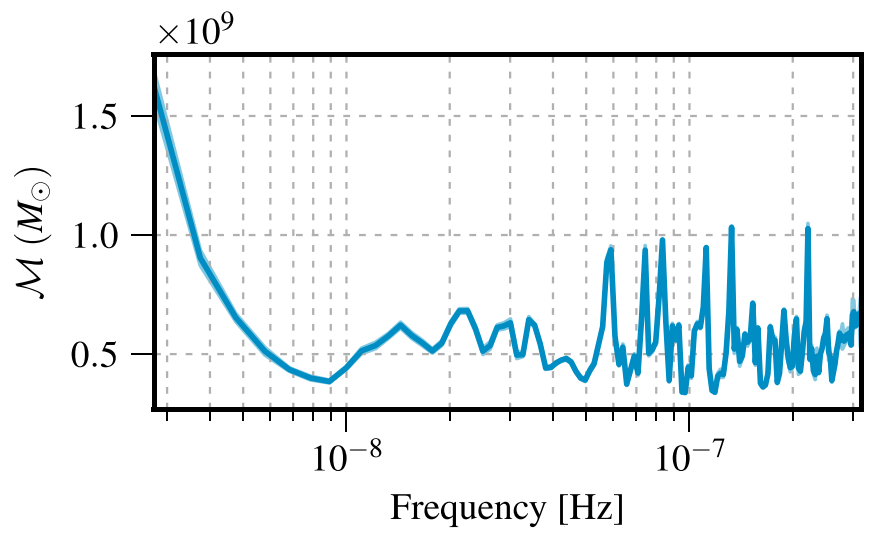

Figure 10. The $95 \%$ upper limit on the chirp mass of any SMBHBs in the Virgo Cluster as a function of GW frequency. We found that there are no SMBHBs in the Virgo Cluster with $\mathcal{M}>1.6(1) \times 10^{9} M_{\odot}$ emitting GWs in this frequency band. At $f_{\mathrm{gw}}=9 \mathrm{nHz}$, we placed an upper limit of 3.8(1) $\times$ $10^{8} M_{\odot}$.

Figure 10 shows the limits on the chirp masses of any SMBHBs in the nearby Virgo Cluster, which is at a distance of 16.5 Mpc. We found that there are no SMBHBs in the Virgo Cluster with $\mathcal{M}>1.6(1) \times 10^{9} M_{\odot}$ emitting GWs in the PTA band. Furthermore, there are no SMBHBs with $\mathcal{M}>3.8(1) \times 10^{8} M_{\odot}$ emitting GWs with $f_{\mathrm{gw}}=9 \mathrm{nHz}$. These chirp-mass limits imply that none of the galaxies NGC 4472 (estimated black hole mass of $2.5 \times 10^{9} M_{\odot}$; Rusli et al. 2013), NGC 4486 (estimated black hole mass of $6.6 \times 10^{9} M_{\odot}$; Gebhardt et al. 2011), or NGC 4649 (estimated black hole mass of $4.5 \times 10^{9} M_{\odot}$; Shen \& Gebhardt 2010) could contain binaries emitting GWs in this frequency range.

In order to assess how likely we were to have detected a SMBHB given our current sensitivity, we compared our strain upper limit curves to simulations of nearby SMBHBs. A similar technique was introduced in Babak et al. (2016) to estimate the detection probability from the strain upper limit curve. We used simulated populations of SMBHBs from Mingarelli et al. (2017), which are based on galaxies in the Two Micron All-Sky Survey (Skrutskie et al. 2006) and merger rates from the Illustris cosmological simulation project (Genel et al. 2014; Rodriguez-Gomez et al. 2015). We estimated the number of detectable sources as the number lying above our sky-averaged 95\% strain upper limit curve. Figure 11 shows the loudest GW sources for a sample realization, plotted alongside our $95 \%$ strain upper limit curve. We show both the sky-averaged strain upper limit curve (solid, blue line) and the strain upper limit curve at the most sensitive sky location (dashed, red line). For this particular simulation, none of the sources were above the sky-averaged strain upper limit curve; therefore, we concluded there were no detectable sources in this particular realization. Out of 75,000 realizations of the local universe, 34 contained a source that lay above the sky-averaged strain upper limit curve (i.e., 0.045\% of realizations contained an observable SMBHB), from which we concluded that our non-detection was unsurprising given our current sensitivity. We point out, though, that our sensitivity varies significantly with sky location, therefore some sources that are below the sky-averaged strain upper limit curve may be detectable depending on their sky locations. In our simulations, we found that a GW source lay above the strain upper limit curve at the most sensitive sky location in 918 realizations $(1.22 \%)$.

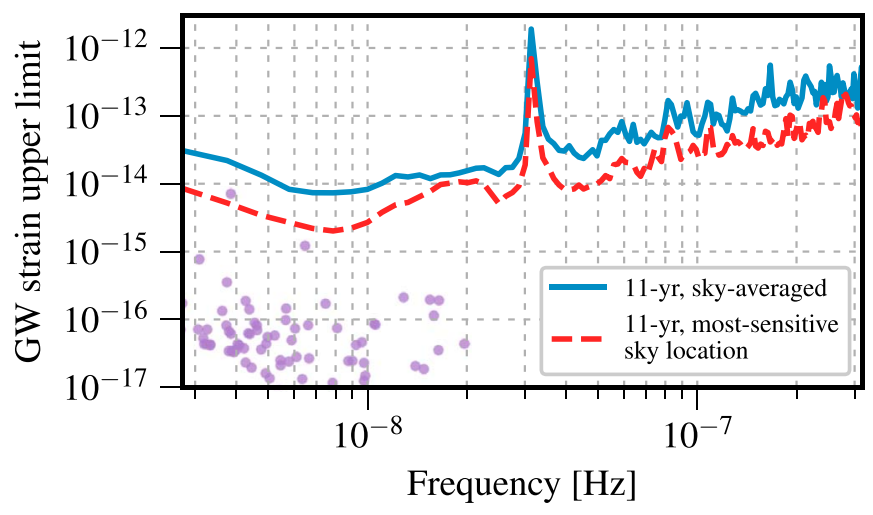

Figure 11. GW frequency and strain for the loudest GW sources for a sample realization of the local universe, plotted alongside our $95 \%$ strain upper limit curve. This simulation used simulated populations of nearby SMBHBs from Mingarelli et al. (2017) to determine the number of sources emitting GWs in the PTA band. For this realization, there are 87 SMBHBs-none of them lie above the sky-averaged strain upper limit curve, and there is one source that lies above the strain upper limit curve at the most sensitive sky location. This source could be detectable depending on its sky location.

\section{Summary and Conclusions}

We searched the NANOGrav $11 \mathrm{yr}$ data set for GWs from individual circular SMBHBs. As we found no strong evidence for GWs in our data, we placed limits on the GW strain. We determined that the $11 \mathrm{yr}$ data set was most sensitive to $f_{\mathrm{gw}}=8 \mathrm{nHz}$, for which the sky-averaged strain upper limit was $h_{0}<7.3(3) \times 10^{-15}$. We produced sky maps of the GW strain upper limit at $f_{\mathrm{gw}}=8 \mathrm{nHz}$. At the most sensitive sky location, we placed a strain upper limit of $h_{0}<$ $2.0(1) \times 10^{-15}$. These results are the first limits on GWs from individual sources to be robust to uncertainties in the SSE due to the incorporation of BAYESEPHEM in our model. As shown in Figure 4, uncertainty in the SSE only affects our sensitivity to GWs for $f_{\mathrm{gw}}<4 \mathrm{nHz}$.

We introduced a new detection technique that uses "dropout" parameters to determine the significance of a common signal in each individual pulsar. We applied this technique to two low-S/N signals found in the $9 \mathrm{yr}$ and $11 \mathrm{yr}$ data sets, and identified the pulsars contributing the most to these signals. This technique is currently being used within NANOGrav in other GW searches, and a methods paper developing this technique is underway. Determining the physical processes causing these low-S/N signals is beyond the scope of this paper. Advanced noise analyses of all the pulsars in the NANOGrav PTA are underway, using more complicated models for the red noise and incorporating models for time variations in the dispersion measure, and the methods and results will be the subject of an upcoming paper.

We used our strain upper limits to place lower limits on the luminosity distance to individual SMBHBs. At the most sensitive sky location, we placed a limit of $d_{L}>120 \mathrm{Mpc}$ for $\mathcal{M}=10^{9} M_{\odot}$ and $d_{L}>5.5 \mathrm{Gpc}$ for $\mathcal{M}=10^{10} M_{\odot}$. Our nondetection of GWs was not surprising given our current sensitivity limits. Using simulated populations of nearby SMBHBs from Mingarelli et al. (2017), we found that only 34 out of 75,000 realizations of the local universe contained a SMBHB whose GW strain lay above our sky-averaged $95 \%$ upper limit curve. These simulations also supported the conclusion that the two low-S/N signals found in the $9 \mathrm{yr}$ and $11 \mathrm{yr}$ data sets were not GW signals. 
Although we have not yet made a positive detection of GWs from individual SMBHBs, the NANOGrav PTA is sensitive enough to place interesting limits on such sources. Based on our non-detection of GWs, we have determined that there are no SMBHBs in the Virgo Cluster with $\mathcal{M}>1.6(1) \times 10^{9} M_{\odot}$ emitting GWs in the PTA band. Furthermore, our sensitivity to GWs from individual SMBHBs will continue to improve as we increase our observation times, add MSPs to our array, and develop improved pulsar noise models.

This paper is the result of the work of dozens of people over the course of more than 13 years. We list specific contributions below. Z.A., K.C., P.B.D., M.E.D., T.D., J.A.E., E.C.F., R.D.F., E.F., P.A.G., G.J., M.L.J., M.T.L., L.L., D.R.L., R.S.L., M.A.M., C.N., D.J.N., T.T.P., S.M.R., P.S.R., R.S., I.H.S., K.S., J.K.S., and W.Z. developed the $11 \mathrm{yr}$ data set. S.J.V. led the search and coordinated the paper writing. J.A.E., S.R.T., P.T.B., S.J.V., and C.A.W. designed and implemented the Bayesian search algorithms in enterprise, and A.R.K., S.T.M., S.R.T., and S.J.V. implemented the $\mathcal{F}_{p}$-statistic in enterprise. N.J.C. helped to uncover a bug in an early implementation of the search algorithms. P.T.B. developed the jump proposals based on empirical distributions. S.J.V., K.I., J.E.T., K.A., P.R.B., A.M.H., and N.S.P. ran the searches. M.R.B, J.A.E., and S.R.T. performed an initial analysis of the $9 \mathrm{yr}$ data set. C.M.F.M., K.I., T.J.W.L., J.S., and S.J.V. did the astrophysical interpretation. S.J.V., K.I., and C.M.F.M. wrote the paper and generated the plots.

We thank the anonymous referee for helpful comments and suggestions that improved this manuscript. The NANOGrav project receives support from National Science Foundation (NSF) Physics Frontier Center award number 1430284. NANOGrav research at UBC is supported by an NSERC Discovery Grant and Discovery Accelerator Supplement and by the Canadian Institute for Advanced Research. Portions of this research were carried out at the Jet Propulsion Laboratory, California Institute of Technology, under a contract with the National Aeronautics and Space Administration. M.V. and J.S. acknowledge support from the JPL RTD program. S.R.T. was partially supported by an appointment to the NASA Postdoctoral Program at the Jet Propulsion Laboratory, administered by Oak Ridge Associated Universities through a contract with NASA. M.A.M., S.B.S., D.R.L., S.T.M., and P.R.B. were supported by NSF award number \#1458952. M.A.M. and N.P. were supported by NSF award number \#1517003. J.A.E. was partially supported by NASA through Einstein Fellowship grants PF4-150120. S.B.S. and C.A.W. were supported by NSF award \#1815664. W.W.Z. is supported by the Chinese Academy of Science Pioneer Hundred Talents Program, the Strategic Priority Research Program of the Chinese Academy of Sciences grant No. XDB23000000, the National Natural Science Foundation of China grant No. 11690024, and by the Astronomical Big Data Joint Research Center, co-founded by the National Astronomical Observatories, Chinese Academy of Sciences and the Alibaba Cloud. Portions of this work performed at N.R.L. are supported by the Chief of Naval Research. The Flatiron Institute is supported by the Simons Foundation.

We thank Logan O'Beirne for helping to uncover a bug in an early implementation of the search algorithms, and for providing comments on the algorithms and manuscript. We thank Casey McGrath for performing some of the CW analyses. We thank Luke Kelley and Tingting Liu for helpful discussions. We thank Alberto Sesana, Xinjiang Zhu, and Siyuan Chen for providing useful comments on the manuscript.

We are grateful for computational resources provided by the Leonard E Parker Center for Gravitation, Cosmology and Astrophysics at the University of Wisconsin-Milwaukee, which is supported by NSF Grants 0923409 and 1626190 . This research made use of the Super Computing System (Spruce Knob) at WVU, which is funded in part by the National Science Foundation EPSCoR Research Infrastructure Improvement Cooperative Agreement \#1003907, the state of West Virginia (WVEPSCoR via the Higher Education Policy Commission) and WVU. This research also made use of the Illinois Campus Cluster, a computing resource that is operated by the Illinois Campus Cluster Program (ICCP) in conjunction with the National Center for Supercomputing Applications (NCSA) and which is supported by funds from the University of Illinois at Urbana-Champaign. Data for this project were collected using the facilities of the Green Bank Observatory and the Arecibo Observatory. The Green Bank Observatory is a facility of the National Science Foundation operated under cooperative agreement by Associated Universities, Inc. The Arecibo Observatory is a facility of the National Science Foundation operated under cooperative agreement by the University of Central Florida in alliance with Yang Enterprises, Inc. and Universidad Metropolitana.

Software: enterprise (Ellis et al. 2017), PAL2 (Ellis \& van Haasteren 2017a), PTMCMCSampler (Ellis \& van Haasteren 2017b).

\section{Appendix \\ Jump Proposals from Empirical Distributions}

In our data analysis pipelines, we computed the posterior distributions using MCMC algorithms, which explore parameter space through a random walk. The $\mathrm{CW}$ search for the $11 \mathrm{yr}$ data set included 154 parameters: 7 common GW parameters, 68 pulsar-term GW parameters, 68 pulsar red-noise parameters, and 11 BAYESEPHEM parameters. Exploring such a large parameter space is computationally intensive, and many iterations are required to burn-in the parameters and ensure the chains have converged. Jump proposals determine how proposed samples are generated, and using particularly good jump proposals can significantly decrease the burn-in and convergence time. Appendix $\mathrm{C}$ of NG5b discusses the jump proposals used in the $\mathrm{CW}$ search of the NANOGrav $5 \mathrm{yr}$ data set, which were also used in the analyses described in this paper.

In the course of analyzing the $11 \mathrm{yr}$ data set, we introduced a new type of jump proposal for the pulsars' red-noise parameters and the BAYESEPHEM parameters. These jump proposals chose new parameter values by drawing from empirical distributions based on the posteriors from an initial Bayesian analysis searching over all of the pulsars that included only the pulsars' red noise and BAYESEPHEM; i.e., excluding any GW term. These jump proposals do not alter the likelihood or the priors - they ensure that the sampler is choosing new parameters that have a high probability of improving the fit, but they do not affect the probability that the new parameter values will be accepted or rejected.

This initial pilot run included only 79 parameters, therefore the red noise and BAYESEPHEM parameters burned-in relatively quickly. We constructed the empirical distributions from histograms of the posteriors, adding one sample to all bins so that the probability density function was nonzero at every point in the prior. For the red-noise parameters, we constructed 
two-dimensional empirical distributions for the amplitude $\log _{10} A$ and spectral index $\gamma$ for each pulsar. For the BAYESEPHEM parameters, we constructed one-dimensional empirical distributions for each of the six Jupiter orbital elements, which describe perturbations to Jupiter's orbit.

We have found that including jumps that draw from the empirical distributions to the MCMC dramatically reduces the number of samples needed for the chains to burn-in and converge because the red noise and BAYESEPHEM parameters converge almost immediately. Efficiently sampling the pulsars' red-noise parameters will become increasingly important as the number of pulsars in our PTA increase, as each pulsar added to the PTA adds two red-noise parameters and two pulsar-term parameters to the model.

\section{ORCID iDs}

P. T. Baker (1) https://orcid.org/0000-0003-2745-753X

S. Burke-Spolaor (1) https://orcid.org/0000-0003-4052-7838

S. Chatterjee (1) https://orcid.org/0000-0002-2878-1502

J. M. Cordes (10) https://orcid.org/0000-0002-4049-1882

N. J. Cornish (1) https://orcid.org/0000-0002-7435-0869

F. Crawford (10 https://orcid.org/0000-0002-2578-0360

H. T. Cromartie (1) https://orcid.org/0000-0002-6039-692X

M. DeCesar (i) https://orcid.org/0000-0002-2185-1790

T. Dolch (1) https://orcid.org/0000-0001-8885-6388

R. D. Ferdman (1) https://orcid.org/0000-0002-2223-1235

P. Gentile (i) https://orcid.org/0000-0001-8158-683X

J. S. Hazboun (1) https://orcid.org/0000-0003-2742-3321

A. M. Holgado (1) https://orcid.org/0000-0003-4143-8132

M. L. Jones (1) https://orcid.org/0000-0001-6607-3710

D. L. Kaplan (1) https://orcid.org/0000-0001-6295-2881

J. S. Key iㅏ https://orcid.org/0000-0003-0123-7600

M. T. Lam (1) https://orcid.org/0000-0003-0721-651X

L. Levin (16 https://orcid.org/0000-0002-2034-2986

D. R. Lorimer (1) https://orcid.org/0000-0003-1301-966X

R. S. Lynch (1) https://orcid.org/0000-0001-5229-7430

D. R. Madison (1) https://orcid.org/0000-0003-2285-0404

M. A. McLaughlin (1) https://orcid.org/0000-0001-7697-7422

C. M. F. Mingarelli (i) https://orcid.org/0000-0002-4307-1322

D. J. Nice (1) https://orcid.org/0000-0002-6709-2566

T. T. Pennucci (ib https://orcid.org/0000-0001-5465-2889

N. S. Pol (1) https://orcid.org/0000-0002-8826-1285

S. M. Ransom (i) https://orcid.org/0000-0001-5799-9714

P. S. Ray (1) https://orcid.org/0000-0002-5297-5278

J. Simon (i) https://orcid.org/0000-0003-1407-6607

R. Spiewak (1) https://orcid.org/0000-0002-6730-3298

I. H. Stairs (i) https://orcid.org/0000-0001-9784-8670

D. R. Stinebring (10) https://orcid.org/0000-0002-1797-3277

K. Stovall (i) https://orcid.org/0000-0002-7261-594X

J. Swiggum (1) https://orcid.org/0000-0002-1075-3837

S. R. Taylor (1) https://orcid.org/0000-0003-0264-1453

J. E. Turner (il https://orcid.org/0000-0002-2451-7288

M. Vallisneri (1) https://orcid.org/0000-0002-4162-0033

R. van Haasteren (1) https://orcid.org/0000-0002-6428-2620

S. J. Vigeland (1) https://orcid.org/0000-0003-4700-9072

C. A. Witt (1) https://orcid.org/0000-0002-6020-9274

W. W. Zhu (1) https://orcid.org/0000-0001-5105-4058

\section{References}

Arzoumanian, Z., Baker, P. T., Brazier, A., et al. 2018b, ApJ, 859, 47 Arzoumanian, Z., Brazier, A., Burke-Spolaor, S., et al. 2014, ApJ, 794, 141
Arzoumanian, Z., Brazier, A., Burke-Spolaor, S., et al. 2015, ApJ, 813, 65 Arzoumanian, Z., Brazier, A., Burke-Spolaor, S., et al. 2016, ApJ, 821, 13 Arzoumanian, Z., Brazier, A., Burke-Spolaor, S., et al. 2018a, ApJS, 235, 37 Babak, S., Petiteau, A., Sesana, A., et al. 2016, MNRAS, 455, 1665 Blanco-Pillado, J. J., Olum, K. D., \& Siemens, X. 2018, PhLB, 778, 392 Burke-Spolaor, S., Taylor, S. R., Charisi, M., et al. 2018, arXiv:1811.08826 Burt, B. J., Lommen, A. N., \& Finn, L. S. 2011, ApJ, 730, 17 Caballero, R. N., Guo, Y. J., Lee, K. J., et al. 2018, MNRAS, 481, 5501 Caprini, C., Durrer, R., \& Siemens, X. 2010, PhRvD, 82, 063511

Chen, S., Middleton, H., Sesana, A., Del Pozzo, W., \& Vecchio, A. 2017, MNRAS, 468, 404

Christy, B., Anella, R., Lommen, A., et al. 2014, ApJ, 794, 163

Corbin, V., \& Cornish, N. J. 2010, arXiv:1008.1782

Cordes, J. M. 2013, CQGra, 30, 224002

Damour, T., \& Vilenkin, A. 2001, PhRvD, 64, 064008

Demorest, P. B., Ferdman, R. D., Gonzalez, M. E., et al. 2013, ApJ, 762, 94 Desvignes, G., Caballero, R. N., Lentati, L., et al. 2016, MNRAS, 458, 3341 Dickey, J. M. 1971, The Annals of Mathematical Statistics, 42, 204

Dolch, T., Lam, M. T., Cordes, J., et al. 2014, ApJ, 794, 21

Efron, B., \& Stein, C. 1981, Anta, 9, 586

Ellis, J., \& van Haasteren, R. 2017a, jellis18/PAL2: PAL2, Zenodo, doi:10. 5281 /zenodo. 251456

Ellis, J., \& van Haasteren, R. 2017b, jellis18/PTMCMCSampler: Official Release, Zenodo, doi:10.5281/zenodo. 1037579

Ellis, J. A., Siemens, X., \& Creighton, J. D. E. 2012, ApJ, 756, 175

Ellis, J. A., Vallisneri, M., Taylor, S. R., \& Baker, P. T. 2017, https://github. com/nanograv/enterprise: enterprise

Folkner, W. M., \& Park, R. S. 2016, JPL planetary and Lunar ephemeris DE436, Tech. rep., Jet Propulsion Laboratory, Pasadena, CA, https://naif. jpl.nasa.gov/pub/naif/JUNO/kernels/spk/de436s.bsp.lbl

Gebhardt, K., Adams, J., Richstone, D., et al. 2011, ApJ, 729, 119

Genel, S., Vogelsberger, M., Springel, V., et al. 2014, MNRAS, 445, 175

Grishchuk, L. P. 2005, PhyU, 48, 1235

Hobbs, G. 2013, CQGra, 30, 224007

Holgado, A. M., Sesana, A., Sandrinelli, A., et al. 2018, MNRAS, 481, L74

Jenet, F. A., Lommen, A., Larson, S. L., \& Wen, L. 2004, ApJ, 606, 799

Jones, M. L., McLaughlin, M. A., Lam, M. T., et al. 2017, ApJ, 841, 125

Kelley, L. Z., Blecha, L., Hernquist, L., Sesana, A., \& Taylor, S. R. 2018, MNRAS, 477, 964

Lam, M. T., Cordes, J. M., Chatterjee, S., et al. 2016, ApJ, 821, 66

Lam, M. T., Cordes, J. M., Chatterjee, S., et al. 2017, ApJ, 834, 35

Lasky, P. D., Mingarelli, C. M. F., Smith, T. L., et al. 2016, PhRvX, 6, 011035

Lee, K. J., Wex, N., Kramer, M., et al. 2011, MNRAS, 414, 3251

Manchester, R. N., Hobbs, G., Bailes, M., et al. 2013, PASA, 30, e017

McLaughlin, M. A. 2013, CQGra, 30, 224008

Middleton, H., Chen, S., Del Pozzo, W., Sesana, A., \& Vecchio, A. 2018, NatCo, 9, 573

Mingarelli, C. M. F., Lazio, T. J. W., Sesana, A., et al. 2017, Nature Astronomy, 1, 886

Ölmez, S., Mandic, V., \& Siemens, X. 2010, Phys. Rev. D, 81, 104028

Rodriguez-Gomez, V., Genel, S., Vogelsberger, M., et al. 2015, MNRAS, 449,49

Rosado, P. A., Sesana, A., \& Gair, J. 2015, MNRAS, 451, 2417

Rusli, S. P., Thomas, J., Saglia, R. P., et al. 2013, AJ, 146, 45

Schutz, K., \& Ma, C.-P. 2016, MNRAS, 459, 1737

Sesana, A. 2013, CQGra, 30, 224014

Sesana, A., Haardt, F., Madau, P., \& Volonteri, M. 2004, ApJ, 611, 623

Sesana, A., Haiman, Z., Kocsis, B., \& Kelley, L. Z. 2018, ApJ, 856, 42

Sesana, A., \& Vecchio, A. 2010, PhRvD, 81, 104008

Shen, J., \& Gebhardt, K. 2010, ApJ, 711, 484

Skrutskie, M. F., Cutri, R. M., Stiening, R., et al. 2006, AJ, 131, 1163

Srivastava, N., Hinton, G., Krizhevsky, A., Sutskever, I., \& Salakhutdinov, R. 2014, Journal of Machine Learning Research, 15, 1929

Taylor, S. R., Huerta, E. A., Gair, J. R., \& McWilliams, S. T. 2016, ApJ, 817,70

Taylor, S. R., Simon, J., \& Sampson, L. 2017, PhRvL, 118, 181102

Tiburzi, C., Hobbs, G., Kerr, M., et al. 2016, MNRAS, 455, 4339

van Haasteren, R., \& Vallisneri, M. 2015, MNRAS, 446, 1170

Verbiest, J. P. W., Lentati, L., Hobbs, G., et al. 2016, MNRAS, 458, 1267

Verbiest, J. P. W., Weisberg, J. M., Chael, A. A., Lee, K. J., \& Lorimer, D. R. 2012, ApJ, 755, 39

Wahlquist, H. 1987, GReGr, 19, 1101

Yardley, D. R. B., Hobbs, G. B., Jenet, F. A., et al. 2010, MNRAS, 407, 669

Zhu, X.-J., Hobbs, G., Wen, L., et al. 2014, MNRAS, 444, 3709 